\title{
Average Current Through a Single-electron Transistor Under Fluctuations of an Observer's Frame of Reference
}

\author{
Yun-Sok Shin \\ Sejong Academy of Science and Arts, Sejong, Republic of Korea \\ Email address: \\ yunsokshin@naver.com

\section{To cite this article:} \\ Yun-Sok Shin. Average Current Through a Single-electron Transistor Under Fluctuations of an Observer's Frame of Reference. American \\ Journal of Physics and Applications. Vol. 7, No. 5, 2019, pp. 118-124. doi: 10.11648/j.ajpa.20190705.14
}

Received: August 13, 2019; Accepted: August 28, 2019; Published: September 16, 2019

\begin{abstract}
The average current through a single-electron transistor (SET) under fluctuations of an observer's frame of reference (OFR) is reported. To date, the average current through a SET has been studied under the assumption that an OFR remains constant throughout the performance of measurements of the current; thus, it remains an unsolved problem as to what is measured of the current when the OFR is assumed to fluctuate. In this paper, a SET comprising a source, drain, and single channel is considered, where an OFR is assumed to be matched to the electrochemical potential energy of the drain of the SET. The average current through the SET for two types of OFR fluctuation is formulated: periodic-square-wave fluctuation and periodic-sawtooth-wave fluctuation, in time representations. Under these types of fluctuation, the average current exhibits a zero-bias Coulomb peak - the amplitude of which gradually increases with the amplitude of the fluctuation type divided by temperature. The amplitude of the zero-bias Coulomb peak is greater in the case of periodic-square-wave fluctuations. Therefore, the amplitude of the zero-bias Coulomb peak together with a varying of both the energy of the channel and the temperature has the potential to reveal the distribution of fluctuations of an OFR.
\end{abstract}

Keywords: Single-electron Transistor, Quantum Dot, Coulomb Peak, Coulomb Blockade, Observer Effect, Fluctuating Frame of Reference

\section{Introduction}

Noise in electronics is a random fluctuation in an electrical signal. Thermal (Johnson-Nyquist) and shot noises are fundamental noises to the operation of a device. Thermal noise $[1,2]$ results from random thermal motion of charge carriers in a device, which occurs at a given temperature $(T)$ regardless of any applied voltage. Shot noise [3] results from statistical fluctuations of an electric current when charge carriers flow across a barrier. Moreover, it is independent of temperature. A classical good conductor exhibits little shot noise as a result of thermally excited charge carriers moving randomly inside the conductor. In contrast, a phase-coherent conductor exhibits much shot noise as a result of diffraction between charge carriers [4], which occurs when the size of the conductor becomes shorter than the electron-phonon scattering length of the charge carriers in the conductor $[5,6]$

A single-electron transistor (SET) [7] is a phase-coherent conductor that provides a platform for single-electron tunneling, such as the Coulomb blockade [7-15] and the spin blockade [16-18], and quantum computation with semiconductor quantum dots (QDs) [19-28]. It comprises a source (electrode), a drain (electrode), two tunnel junctions, and a quantum dot (a conductive island). The electrochemical potential of the island can be tuned by a third electrode, known as a gate, which is capacitively coupled to the island. If the energy of the island is higher than the electrochemical potential energies (EPEs) of the source and drain (that is, it is in the Coulomb blockade regime), there will be no current flowing the channel. Through an application of a positive voltage to the gate, the energy of the island can be aligned to the EPE of the source, thus providing the conditions for an electron in the source (drain) to be able to move toward the drain (source) by sequential transitions between the tunnel junctions via the island. If the electron were to reach the drain (source), an electric current would flow through the SET and the SET would experience noise signals. An electric current through a SET is determined by the difference 
between the EPEs of the source and drain, and is independent of an observer's frame of reference (OFR). Consequently, to date, an electric current through a SET has been studied under the assumption that an OFR remains constantconventionally, the OFR is assumed to be zero throughout the performance of measurements of the current. Thus, it remains an open question as to what is measured of the current when the OFR is assumed to fluctuate.

Recently, a novel observer effect in an Einstein solid, induced by fluctuations of an OFR, has been proposed [29]. The molar specific heat at constant volume of an Einstein solid as a function of temperature would provide clear criteria for characterizing the distribution of fluctuations of an OFR. It would be zero at temperature $T=0 \mathrm{~K}$, but would have a peak at low temperatures for periodic-square-wave fluctuations. In addition, it would converge to $3 R$ for periodic-sawtooth-wave fluctuations, where $R$ is the gas constant.

If the EPE of the drain of a SET is matched to an OFR, then it is possible to investigate an observer effect induced by fluctuations of the OFR. In this study, the average current through a SET comprising a source, drain, and single channel [30] is investigated under fluctuations of an OFR. For pedagogical and experimental demonstrations, the average current is formulated for two types of fluctuation: periodic-

$$
I_{\mathrm{avg}}=\frac{1}{\Delta t} \int_{t_{i}}^{t_{f}}\left[I_{1}(t)-I_{2}(t)\right] d t=\frac{I_{0}}{\Delta t} \int_{t_{i}}^{t_{f}}\left[f_{1}(\varepsilon, t)-f_{2}(\varepsilon, t)\right] d t
$$

where $I_{j}(t)$ is the current through the SET flowing from $\mu_{j}$ at time $t$ and $I_{0}=\frac{2 q_{0}}{\hbar} \frac{\gamma_{1} \gamma_{2}}{\gamma_{1}+\gamma_{2}}$ and $f_{j}(\varepsilon, t)=[1+\exp ((\varepsilon-$ $\left.\left.\left.\mu_{j}(t)\right) / k T\right)\right]^{-1}$ is the Fermi-Dirac distribution for $j=1,2$. Here, "1" and "2" denote the "left (source)" and "right (drain)" electrodes, respectively. In this study, the charge of an electron, $q_{0}$, is assumed to be positive (for simplicity); $\hbar$ is the Dirac constant; $k$ is the Boltzmann constant; and $t_{i}$ and $t_{f}$ are the initial and final times measured by the observer, respectively.

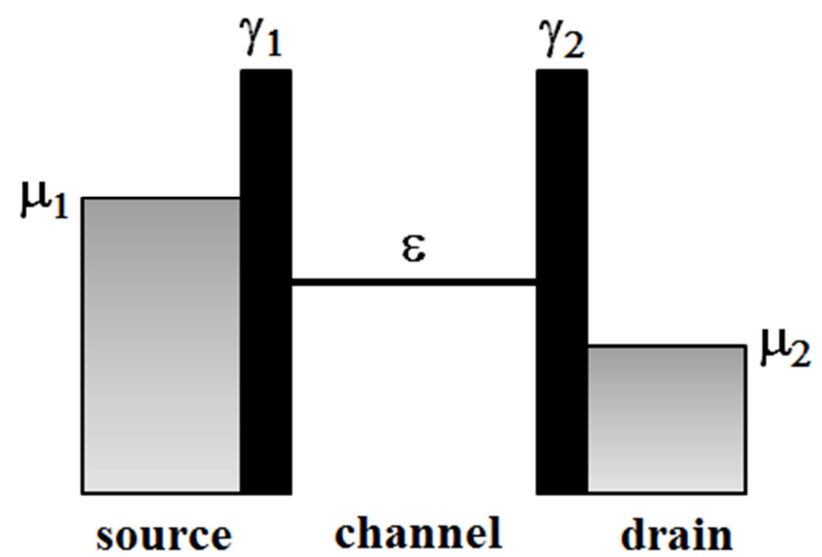

Figure 1. Schematic of a single-electron transistor (SET) comprising a source, drain, and single channel, where $\mu_{1}$ and $\mu_{2}$ are the electrochemical potential energies of the left (source) and right (drain) electrodes, respectively; $\varepsilon$ the energy of the channel; and $\gamma_{1}$ and $\gamma_{2}$ the source-channel and drain-channel tunneling rates, respectively. An electric current through the SET is monitored by an observer connected to the drain. square-wave fluctuation and periodic-sawtooth-wave fluctuation, in time representations. Under these types of fluctuation, a zero-bias Coulomb peak appears in the average current, which is asymmetric as a function of the energy of the channel divided by temperature. The amplitude of the peak monotonically increases with the amplitude of the fluctuation type divided by temperature. Therefore, the amplitude of the zero-bias Coulomb peak together with a varying of both the energy of the channel and the temperature would reveal the distribution of fluctuations of an OFR.

\section{Average Current Through a SET with a Single Channel}

Figure 1 shows a schematic energy diagram of a SET with a single channel, where $\mu_{1(2)}$ is the EPE of the left (right) electrode, $\varepsilon$ the energy of the channel, $\gamma_{1(2)}$ the tunneling rate between the left (right) electrode and channel [30]. An electric current through the channel is monitored by an observer, where the OFR is matched to the EPE of the right electrode (the drain). For the energy of an observer with fluctuating frame of reference at time $t, E_{\text {ref }}(t)$, the average current with time interval $\Delta \mathrm{t}\left(=t_{f}-t_{i}\right), I_{\mathrm{avg}}$, can be expressed as

\subsection{Fluctuating Drain}

Given $\mu_{1}(t)=\mu_{1}$ and $\mu_{2}(t)=\mu_{2}-E_{\text {ref }}(t)$ (figure 2), $I_{\text {avg }}$ can be expressed as

$$
I_{\mathrm{avg}}=\frac{I_{0}}{1+\exp \left[\left(\varepsilon-\mu_{1}\right) / k T\right]}-\int_{t_{i}}^{t_{f}} \frac{I_{0}}{1+\exp \left[\left(\varepsilon-\mu_{2}(t)\right) / k T\right]} d t
$$

(a)

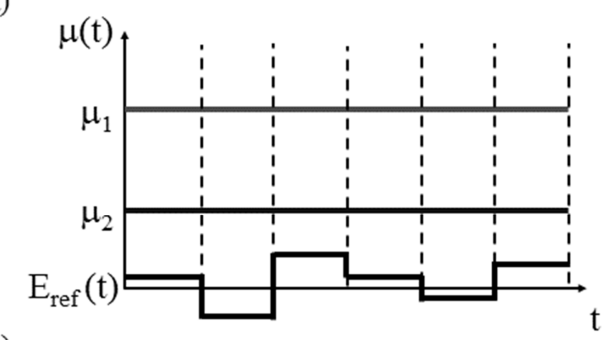

(b)

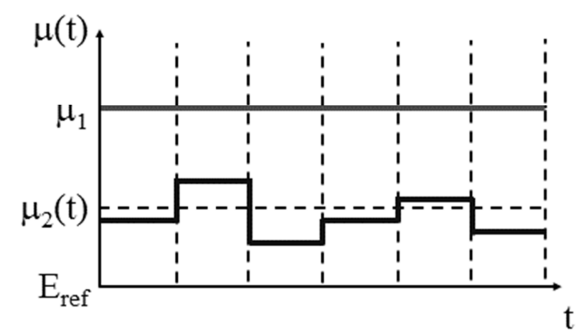

Figure 2. Schematic energy diagram of a SET comprising a source, drain, and single channel. (a) Stationary electrochemical potential energies, $\mu_{1}$ and $\mu_{2}$, and fluctuating frame of reference at time $t, E_{\text {ref }}(t)$. (b) Measured energy for $\mu_{2}$ at time $t, \mu_{2}(t)\left(=\mu_{2}-E_{\text {ref }}(t)\right)$, and stationary frame of reference, $E_{\text {ref }}$, which is taken to be equal to 0 . 


\subsection{Stationary Drain}

For an observer with a stationary frame of reference $\left(E_{\mathrm{ref}}=0\right)$, the corresponding current $I_{1}$ is expressed as

$$
I_{1}=\frac{I_{0}}{1+\exp \left[\left(\varepsilon-\mu_{1}\right) / k T\right]}-\frac{I_{0}}{1+\exp \left[\left(\varepsilon-\mu_{2}\right) / k T\right]}
$$

From $d I_{1} / d \varepsilon=0$, it is found that $I_{1}$ has a minimum or maximum (labeled a "Coulomb peak") at $\varepsilon=\left(\mu_{1}+\mu_{2}\right) / 2$. At low-bias limits of $\mu_{1} \ll k T$, the differential current at the peak is $\Delta I_{1}=I_{1}\left(\mu_{1}\right)-I_{1}(0) \approx I_{0} \mu_{1} /\left[4 k T \cosh ^{2}(\varepsilon / 2 k T)\right]$ and is proportional to $\mu_{1}$. At high-bias limits of $\mu_{1} \gg k T$, the Coulomb peak is located at $\varepsilon=\mu_{1} / 2$, where $\mu_{2}=0$.

The differential conductance, $G_{1}$, can be expressed as

$$
G_{1}=q_{0} d I_{1} / d \mu_{1}=\frac{q_{0} I_{0}}{4 k T \cosh ^{2}\left[\left(\varepsilon-\mu_{1}\right) / 2 k T\right]}
$$

From $d G_{1} / d \mu_{1}=0$, it is found that $G_{1}$ has a Coulomb peak at $\varepsilon=\mu_{1}$.

(a)

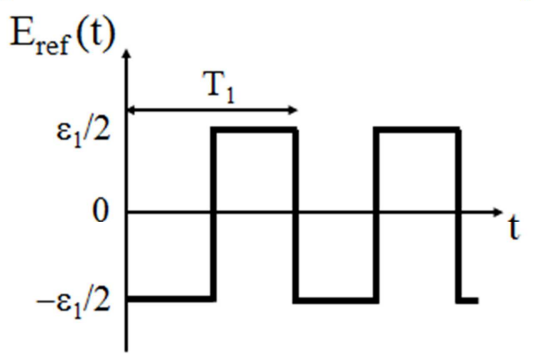

\section{Average Current Through a SET Under Periodic-square-wave Fluctuations}

Given periodic

$$
\mathrm{E}_{\mathrm{ref}}(t)=\left\{\begin{array}{l}
-\varepsilon_{1} / 2 \text { for } 0 \leq t<T_{1} / 2 \\
\varepsilon_{1} / 2 \text { for } T_{1} / 2 \leq t<T_{1}
\end{array}\right.
$$

[figure 3(a)], the measured EPE for $\mu_{2}$ at time $t, \mu_{2}(t)$, can be expressed as

$$
\mu_{2}(t)=\left\{\begin{array}{l}
\mu_{2}+\varepsilon_{1} / 2 \text { for } 0 \leq t<T_{1} / 2 \\
\mu_{2}-\varepsilon_{1} / 2 \text { for } T_{1} / 2 \leq t<T_{1}
\end{array}\right.
$$

where $\varepsilon_{1} / 2$ and $T_{1}$ are the amplitude and period of the periodic-square-wave fluctuations, respectively.

(b)

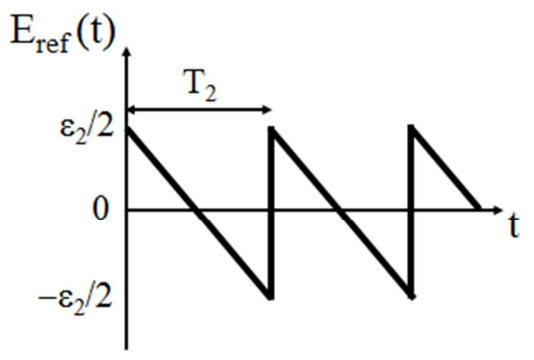

Figure 3. (a) Fluctuating frame of reference at time $t, E_{r e f}(t)$, for periodic-square-wave fluctuations, of which the amplitude and period are $\varepsilon_{1} / 2$ and $T_{1}$, respectively. (b) Fluctuating frame of reference at time $t, E_{\text {ref }}(t)$, for periodic-sawtooth-wave fluctuations, of which the amplitude and period are $\varepsilon_{2} / 2$ and $T_{2}$, respectively.

\subsection{Periodic-square-wave Fluctuations of a Half Period}

For an observer with fluctuating frame of reference by means of half-period periodic-square-wave fluctuations, the corresponding current, $I_{2}$, is expressed as

$$
I_{2}=\frac{I_{0}}{1+\exp \left[\left(\varepsilon-\mu_{1}\right) / k T\right]}-\frac{I_{0}}{1+\exp \left[\left(\varepsilon-\mu_{2}-\varepsilon_{1} / 2\right) / k T\right]}
$$

where $t_{i}=0$ and $t_{f}=T_{1} / 2$.

$$
\begin{gathered}
I_{2}=\frac{I_{0}}{1+\exp \left[\left(\varepsilon-\mu_{1}\right) / k T\right]}-\frac{I_{0}}{1+\exp \left[\left(\varepsilon-\mu_{2}+\varepsilon_{1} / 2\right) / k T\right]} \\
I_{3}=\frac{I_{0}}{1+\exp \left[\left(\varepsilon-\mu_{1}\right) / k T\right]}-\frac{I_{0} / 2}{1+\exp \left[\left(\varepsilon-\mu_{2}-\varepsilon_{1} / 2\right) / k T\right]}-\frac{I_{0} / 2}{1+\exp \left[\left(\varepsilon-\mu_{2}+\varepsilon_{1} / 2\right) / k T\right]}
\end{gathered}
$$

where $t_{i}=T_{1} / 2$ and $t_{f}=T_{1}$. From $d I_{2} / d \varepsilon=0$, it is found that $I_{2}$ has a Coulomb peak at $\varepsilon=\left(\mu_{1}+\mu_{2} \pm \varepsilon_{1} / 2\right) / 2$. The differential conductance $G_{2}=q_{0} d I_{2} / d \mu_{1}=G_{1}$ is independent of the fluctuations on the drain.

\subsection{Periodic-square-wave Fluctuations of One Period}

For an observer with fluctuating frame of reference by means of one-period periodic-square-wave fluctuations, the corresponding average current of the SET, $I_{3}$, is expressed as where $t_{i}=0$ and $t_{f}=T_{1}$.

In figure $4(\mathrm{a}), I_{3} / I_{0}$ is displayed as a function of $\varepsilon / k T$ at various $\varepsilon_{1} / k T$, where $\mu_{1}=\mu_{2}=0$. A zero-bias Coulomb peak is located at $\varepsilon / k T= \pm 1.317$ at $\varepsilon_{1} / k T=0.01$. The absolute value of $\varepsilon / k T,|\varepsilon / k T|$, and the amplitude of the peak monotonically increase with $\varepsilon_{1} / k T$. For $0<t<T_{1} / 2$ and $\varepsilon>0$, the energy of the channel is greater than the EPE of the source but less than that of the drain. Consequently, there is a current flowing through the channel from the drain (right) to the source (left), which, in turn, means there is movement of electrons between the source and the drain. The presence of a current flowing through the channel results in a negative zero-bias Coulomb peak (figure 4(a), upper inset). For $T_{1} /$ $2<t<T_{1}$ and $\varepsilon<0$, the energy of the channel is greater than the EPE of the drain but less than that of the source. Consequently, there is a current flowing through the channel from the source (left) to the drain (right), which, in turn, means there is movement of electrons between the source and the drain. The presence of a current flowing through the channel results in a positive zero-bias Coulomb peak (figure 4(a), lower inset).

From $d I_{3} / d \varepsilon=0$, it is found that a zero-bias Coulomb 
peak is located at $\varepsilon / k T$, expressed as

$$
\cosh (\varepsilon / k T)=\frac{1+\sqrt{1+4\left(1+\cosh \left(\varepsilon_{1} / 2 k T\right)\right)}}{2}
$$

where $\mu_{1}=\mu_{2}=0$. According to (9), the peak is located at $|\varepsilon / k T|=\cosh ^{-1}(2)=1.317$ in the limits of $\varepsilon_{1} / k T \rightarrow 0$ and the absolute value of $\varepsilon / k T,|\varepsilon / k T|$, monotonically increases with $\varepsilon_{1} / k T$. The positive zero-bias Coulomb peak located at $\varepsilon / k T=-1.317$ is in the limit of $\mu_{1} / k T \rightarrow 0$, and this value of $\varepsilon / k T$ of the peak increases with increasing $\mu_{1} / k T$. At low-bias limits of $\mu_{1} \ll k T$, the differential current at the peak is $\Delta I_{3}=I_{3}\left(\mu_{1}\right)-I_{3}(0) \approx$ $I_{0} \mu_{1} /\left[4 k T \cosh ^{2}(\varepsilon / 2 k T)\right]$ and is proportional to $\mu_{1}$ [figure 4(b)]. The peak is located at $\varepsilon=\mu_{1} / 2$ at high-bias limits of $\mu_{1} \gg k T$, which agrees with that of $I_{1}$. The differential conductance $G_{3}=q_{0} d I_{3} / d \mu_{1}=G_{1}$ is independent of the fluctuations on the drain.

On the other hand, a zero-bias Coulomb peak can be induced by a thermoelectric effect in a mesoscopic device, such as a molecular device [31] or SET, with temperature difference between two electrodes [32]. Regarding a SET, the electric current through a SET is expressed as $I_{1}{ }^{\prime}=I_{0} /[1+$ $\left.\exp \left[\left(\varepsilon-\mu_{1}\right) / k T_{1}\right]\right]-I_{0} /\left[1+\exp \left[\left(\varepsilon-\mu_{2}\right) / k T_{2}\right]\right]$, where $T_{1}$ and $T_{2}$ are temperatures of the left (source) and right (drain) electrodes, respectively. From $\mathrm{dI}_{1}{ }^{\prime} / d \varepsilon=0$, it is found that a zero-bias Coulomb peak is located at $\varepsilon$,

(a)

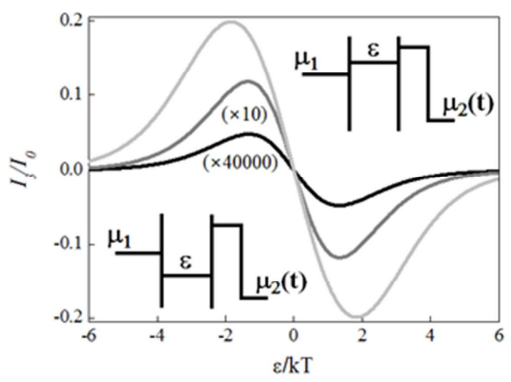

(b)

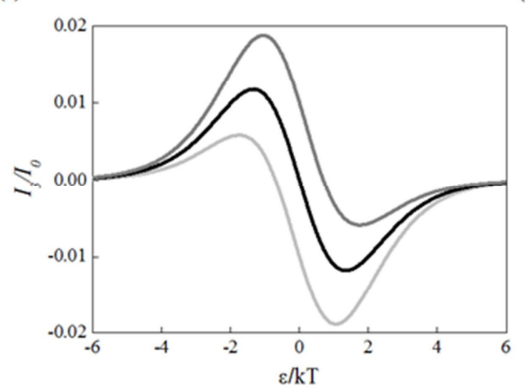

expressed as $\frac{\cosh \left(\varepsilon / 2 k T_{1}\right)}{\cosh \left(\varepsilon / 2 k T_{2}\right)}=\sqrt{\frac{T_{2}}{T_{1}}}$, where $\mu_{1}=\mu_{2}=0$. From numerical calculations, the zero-bias Coulomb peak is located at the absolute value of $\varepsilon / k T_{1},\left|\varepsilon / k T_{1}\right|=1.5434$, where $T_{1}=1 \mathrm{~K}$ and $T_{2}=1.00001 \mathrm{~K}$ and the absolute value of $\varepsilon / k T_{1},\left|\varepsilon / k T_{1}\right|$, monotonically increases with temperature difference between $T_{1}$ and $T_{2}, \Delta T\left(=T_{2}-T_{1}\right)$. The positive zero-bias Coulomb peak located at $\varepsilon / k T_{1}=-1.5434$ is in the limit of $\mu_{1} / k T \rightarrow 0$, and this value of $\varepsilon / k T$ of the peak increases with increasing $\mu_{1} / k T$. At low-bias limits of $\mu_{1} \ll$ $k T_{1}$, the differential current, $\Delta I_{1}{ }^{\prime}$, can be expressed as $\Delta I_{1}{ }^{\prime}=$ $I_{1}{ }^{\prime}\left(\mu_{1}\right)-I_{1}{ }^{\prime}(0) \approx I_{0} \mu_{1} /\left[4 k T_{1} \cosh ^{2}\left(\varepsilon / 2 k T_{1}\right)\right]$.

For confirmation, $I_{1}^{\prime} / I_{0}$ is displayed as a function of $\varepsilon / k T_{1}$ at various $T_{2}$ in figure $4(\mathrm{c})$, where $T_{1}=1 \mathrm{~K}$ and $\mu_{1}=\mu_{2}=0$. Whether a zero-bias Coulomb peak is positive or not depends on only the direction of $\Delta T$. In a configuration where the left and right electrodes are used as the drain and source, respectively, the electric current and the resulting zero-bias Coulomb peak are reversed from those in the previous configuration because $\Delta T$ is reversed. In contrast to a thermoelectric effect, the average current under the fluctuations on the drain is strongly dependent on $\Delta \mathrm{t}$ but constant to the operation configurations. In this regard, a zero-bias Coulomb peak induced by a thermoelectric effect could be distinguished from that induced by fluctuations on the drain.

(c)

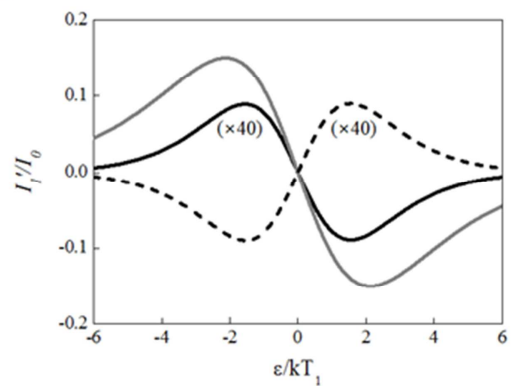

Figure 4. (a) $I_{3} / I_{0}$ as a function of $\varepsilon / k T$ at various $\varepsilon_{1} / k T$, where $\mu_{1}=\mu_{2}=0$. Here, $\varepsilon_{1} / k T=5$ (light gray), 1 (gray), and 0.01 (black). Note that the data at $\varepsilon_{1} / k T=1$ and 0.01 are exaggerated by a factor of 10 and 40,000, respectively, for clarity. The lower (upper) inset shows a schematic energy diagram for the positive (negative) Coulomb peak under periodic-square-wave fluctuations of one period. (b) $I_{3} / I_{0}$ as a function of $\varepsilon / k T$ at various $\mu_{1} / k T$, where $\varepsilon_{1} / k T=1$. Here, $\mu_{1} / k T=0.04$ (gray), 0 (black), and -0.04 (light gray). (c) $I_{1}^{\prime} / I_{0}$ as a function of $\varepsilon / k T_{1}$ at various $T_{2}$, where $T_{1}=1 \mathrm{~K}$ and $\mu_{1}=\mu_{2}=0$. Here, $T_{2}=1.01 \mathrm{~K}$ (black), $2 \mathrm{~K}$ (gray), and $0.99 \mathrm{~K}$ (dotted black). Note that the data at $T_{2}=1.01 \mathrm{~K}$ and $0.99 \mathrm{~K}$ are exaggerated by a factor of 40 for clarity.

\subsection{Periodic-square-wave Fluctuations at High-frequency Limits}

means of periodic-square-wave fluctuations at highfrequency limits, the corresponding average current of the SET, $I_{3}{ }^{\prime}$, is expressed as

For an observer with fluctuating frame of reference by

$$
I_{3}{ }^{\prime} \approx \frac{I_{0}}{1+\exp \left[\left(\varepsilon-\mu_{1}\right) / k T\right]}-\frac{I_{0} / 2}{1+\exp \left[\left(\varepsilon-\mu_{2}-\varepsilon_{1} / 2\right) / k T\right]}-\frac{I_{0} / 2}{1+\exp \left[\left(\varepsilon-\mu_{2}+\varepsilon_{1} / 2\right) / k T\right]}=I_{3}
$$

where $t_{i}=0$ and $t_{f} \gg T_{1}$

\section{Average Current Through a SET Under Periodic-sawtooth-wave Fluctuations}

Given periodic $\mathrm{E}_{\mathrm{ref}}(t)=\frac{\varepsilon_{2}}{2}-\frac{\varepsilon_{2}}{T_{2}} t$ for $0 \leq t<T_{2}$ [figure 3(b)], the measured EPE for $\mu_{2}$ at time $t, \mu_{2}(t)$, can be expressed as

$$
\mu_{2}(t)=\mu_{2}+\frac{\varepsilon_{2}}{T_{2}} t-\frac{\varepsilon_{2}}{2} \text { for } 0 \leq t<T_{2},
$$

where $\varepsilon_{2} / 2$ and $T_{2}$ are the amplitude and period of the periodic-sawtooth-wave fluctuations, respectively. 


\subsection{Periodic-sawtooth-wave Fluctuations of One Period}

For an observer with fluctuating frame of reference by means of one-period periodic-sawtooth-wave fluctuations, the corresponding average current of the SET, $I_{4}$, is expressed as

$$
I_{4}=\frac{I_{0}}{1+\exp \left[\left(\varepsilon-\mu_{1}\right) / k T\right]}-I_{0}-I_{0} \frac{k T}{\varepsilon_{2}} \ln \left[\frac{1+\exp \left[\left(\varepsilon-\mu_{2}-\varepsilon_{2} / 2\right) / k T\right]}{1+\exp \left[\left(\varepsilon-\mu_{2}+\varepsilon_{2} / 2\right) / k T\right]}\right]
$$

In figure 5(a), $I_{4} / I_{0}$ is displayed as a function of $\varepsilon / k T$ at various $\varepsilon_{2} / k T$, where $\mu_{1}=\mu_{2}=0$. A zero-bias Coulomb peak is located at $\varepsilon / k T= \pm 1.317$ at $\varepsilon_{2} / k T=0.01$ and the absolute value of $\varepsilon / k T,|\varepsilon / k T|$, and the amplitude of the peak monotonically increase with $\varepsilon_{2} / k T$.

From $d I_{4} / d \varepsilon=0$, it is found that a zero-bias Coulomb peak is located at $\varepsilon / k T$, expressed as

$$
\exp (|\varepsilon / k T|)=\frac{\sinh \left(z_{0}\right)-z_{0} \cosh \left(z_{0}\right)-\sqrt{z_{0} \sinh \left(z_{0}\right)\left(z_{0} \sinh \left(z_{0}\right)-2 \cosh \left(z_{0}\right)+2\right)}}{z_{0}-\sinh \left(z_{0}\right)}
$$

where $z_{0}=\varepsilon_{2} / 2 k T$. According to (13), the peak is located at $|\varepsilon / k T|=\ln (2+\sqrt{3})=1.317$ in the limits of $\varepsilon_{2} / k T \rightarrow 0$, and the absolute value of $\varepsilon / k T,|\varepsilon / k T|$, monotonically increases with $\varepsilon_{2} / k T$. The positive zero-bias Coulomb peak located at $\varepsilon / k T_{1}=-1.317$ is in the limit of $\mu_{1} / k T \rightarrow 0$, and this value of $\varepsilon / k T$ of the peak increases with increasing $\mu_{1} / k T$. At low-bias limits of $\mu_{1} \ll k T$, the

(a)

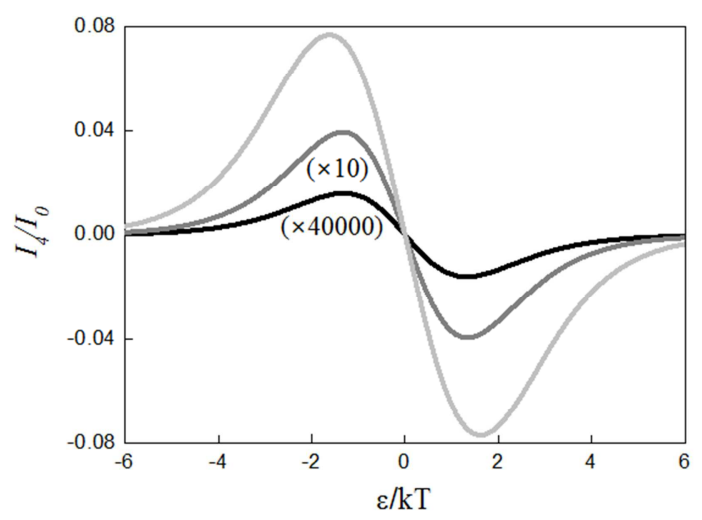

differential current at the peak is $\Delta I_{4}=I_{4}\left(\mu_{1}\right)-I_{4}(0) \approx$ $I_{0} \mu_{1} /\left[4 k T \cosh ^{2}(\varepsilon / 2 k T)\right]$ and is proportional to $\mu_{1}$ [figure $5(\mathrm{~b})]$. The peak is located at $\varepsilon=\mu_{1} / 2$ at high-bias limits of $\mu_{1} \gg k T$, which also agrees with that of $I_{1}$. The differential conductance $G_{4}=q_{0} d I_{4} / d \mu_{1}=G_{1} \quad$ is independent of the fluctuations on the drain.

(b)

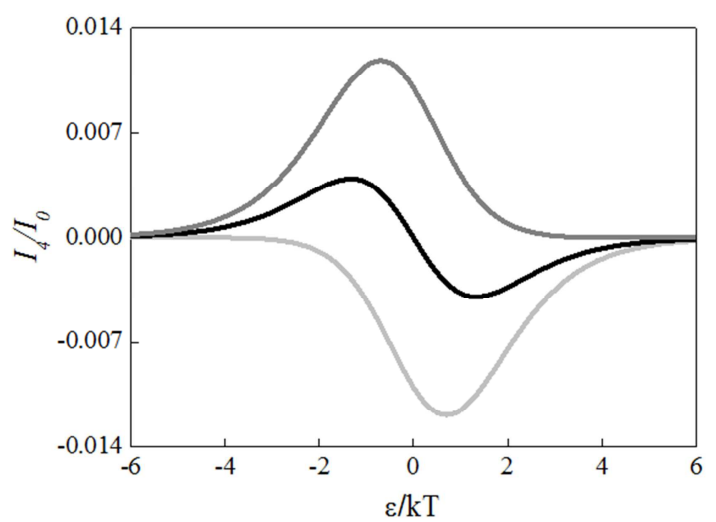

Figure 5. (a) $I_{4} / I_{0}$ as a function of $\varepsilon / k T$ at various $\varepsilon_{2} / k T$, where $\mu_{1}=\mu_{2}=0$. Here, $\varepsilon_{2} / k T=5$ (light gray), 1 (gray), and 0.01 (black). Note that the data at $\varepsilon_{2} / k T=1$ and 0.01 are exaggerated by a factor of 10 and 40,000 , respectively, for clarity. (b) $I_{4} / I_{0}$ as a function of $\varepsilon / k T$ at various $\mu_{1} / k T$, where $\varepsilon_{2} / k T=1$. Here, $\mu_{1} / k T=0.04$ (gray), 0 (black), and -0.04 (light gray).

\subsection{Periodic-sawtooth-wave Fluctuations at High-frequency Limits}

For an observer with fluctuating frame of reference by means of periodic-sawtooth-wave fluctuations at highfrequency limits, the corresponding average current of the SET, $I_{4}{ }^{\prime}$, is expressed as

$$
I_{4}{ }^{\prime} \approx \frac{I_{0}}{1+\exp \left[\left(\varepsilon-\mu_{1}\right) / k T\right]}-I_{0}-I_{0} \frac{k T}{\varepsilon_{2}} \ln \left[\frac{1+\exp \left[\left(\varepsilon-\mu_{2}-\varepsilon_{2} / 2\right) / k T\right]}{1+\exp \left[\left(\varepsilon-\mu_{2}+\varepsilon_{2} / 2\right) / k T\right]}\right]=I_{4}
$$

where $t_{i}=0$ and $t_{f} \gg T_{1}$.

\section{Conclusions}

In this paper, a zero-bias Coulomb peak in the average current through a single-electron transistor (SET) comprising a source, drain, and single channel is shown under periodic fluctuations of an observer's frame of reference (OFR). The energy value and amplitude of the peak is quantitatively calculated. Moreover, the average current through the SET is formulated for two types of fluctuation — periodic-square- wave fluctuation and sawtooth-wave fluctuation, in time representations - on the drain, the electrochemical potential energy of which is matched to the OFR. Under periodicsquare-wave fluctuations of one period or at high-frequency limits, the average current exhibits a zero-bias Coulomb peak, the amplitude of which gradually increases with the amplitude of the fluctuations. Similarly, under periodicsawtooth-wave fluctuations of one period or at highfrequency limits, the average current exhibits a zero-bias Coulomb peak, but the amplitude of the peak in this case is smaller than in the case of periodic-square-wave fluctuations. 
Accordingly, a zero-bias Coulomb peak in an average current through a SET with a varying energy of the channel of the SET divided by temperature would provide clear criteria for characterizing the distribution of fluctuations of an OFR.

\section{Acknowledgements}

This work was supported by Sejong Academy of Science and Arts.

\section{References}

[1] J. Johnson, "Thermal agitation of electricity in conductors," Phys. Rev., vol. 32, pp. 97-109, 1928.

[2] H. Nyquist, "Thermal agitation of electric charge in conductors," Phys. Rev., vol. 32, pp. 110-113, 1928.

[3] W. Schottky, "Über spontane Stromschwankungen in verschiedenen Elektrizitätsleitern," Ann. Phys. (Leipzig), vol. 362, pp. 541-567, 1918.

[4] C. W. J. Beenakker and H. van Houten, "Semiclassical theory of shot noise and its suppression in a conductor with deterministic scattering,” Phys. Rev. B, vol. 43, pp. 12066-12069, 1991.

[5] A. H. Steinbach, J. M. Martinis, and M. H. Devoret, "Observation of hot-electron shot noise in a metallic resistor," Phys. Rev. Lett., vol. 76, pp. 3806-3809, 1996.

[6] Ya. M. Blanter and M. Büttiker, "Shot noise in mesoscopic conductors," Phys. Rep., vol. 336, pp. 1-166, 2000.

[7] D. V. Averin and K. K. Likharev, "Coulomb blockade of singleelectron tunneling, and coherent oscillations in small tunnel junctions," J. Low Temp. Phys. Rep., vol. 62, pp. 345-373, 1986.

[8] P. L. McEuen, E. B. Foxman, J. M. Kinaret, U. Meirav, M. A. Kastner, N. S. Wingreen, and S. J. Wind, "Self-consistent addition spectrum of a Coulomb island in the quantum Hall regime," Phys. Rev. B, vol. 45, pp. 11419-11422, 1992.

[9] E. Leobandung, L. Guo, Y. Wang, and S. Y. Chou, "Observation of quantum effects and Coulomb blockade in silicon quantum-dot transistors at temperatures over $100 \mathrm{~K}$," Appl. Phys. Lett., vol. 67, pp. 938-940, 1995.

[10] S. Tarucha, D. G. Austing, T. Honda, R. J. van der Hage, and L. P. Kouwenhoven, "Shell filling and spin effects in a few electron quantum dot," Phys. Rev. Lett., vol. 77, pp. 3613$3616,1996$.

[11] L. P. Kouwenhoven, T. H. Oosterkamp, M. W. S. Danoesastro, M. Eto, D. G. Austing, T. Honda, and S. Tarucha, "Excitation spectra of circular, few-electron quantum dots," Science, vol. 278, pp. 1788-1792, 1997.

[12] L. Gaudreau, A. Kam, G. Granger, S. A. Studenikin, P. Zawadzki, and A. S. Sachrajda, "A tunable few electron triple quantum dot,” Appl. Phys. Lett., vol. 95, pp. 193101, 2009.

[13] H. Flentje, B. Bertrand, P.-A. Mortemousque, V. Thiney, A. Ludwig, A. D. Wieck, C. Bäuerle, and T. Meunier, "A linear triple quantum dot system in isolated configuration," Appl. Phys. Lett., vol. 110, pp. 233101, 2017.

[14] A. Noiri, K. Kawasaki, T. Otsuka, T. Nakajima, J. Yoneda, S. Amaha, M. R. Delbecq, K. Takeda, G. Allison, A. Ludwig, A.
D. Wieck, and S. Tarucha, "A triangular triple quantum dot with tunable tunnel couplings," Sem. Sci. Tech., vol. 32, pp. 084004, 2017.

[15] C. Hong, G. Yoo, J. Park, M.-K. Cho, Y. Chung, H.-S. Sim, D. Kim, H. Choi, V. Umansky, and D. Mahalu, "Attractive Coulomb interactions in a triple quantum dot," Phys. Rev. B, vol. 97, pp. 241115, 2018.

[16] K. Ono, D. G. Austing, Y. Tokura, and S. Tarucha, "Current rectification by Pauli exclusion in a weakly coupled double quantum dot system," Science, vol. 297, pp. 1313-1317, 2002.

[17] H. W. Liu, T. Fujisawa, Y. Ono, H. Inokawa, A. Fujiwara, K. Takashina, and Y. Hirayama, "Pauli-spin-blockade transport through a silicon double quantum dot," Phys. Rev. B, vol. 77, pp. 073310, 2008.

[18] D. Kotekar-Patil. A. Corna, R. Maurand, A. Crippa, A. Orlov, S. Barraud, L. Hutin, M. Vinet, X. Jehl, S. De Dranceschi, and M. Sanquer, "Pauli spin blockade in CMOS double quantum dot devices," Phys. Stat. Sol. (b), vol. 254, pp. 201600581, 2016.

[19] D. Loss and D. P. DiVincenzo, "Quantum computation with quantum dots,” Phys. Rev. A, vol. 57, pp. 120-126, 1998.

[20] L. M. K. Vandersypen, J. M. Elzerman, R. N. Schouten, L. H. Willems van Beveren, R. Hanson, and L. P. Kouwenhoven, "Real-time detection of single-electron tunneling using a quantum point contact," Appl. Phys. Lett., vol. 85, pp. 4394 4396, 2004.

[21] J. M. Elzerman, R. Hanson, L. H. Willems van Beveren, B. Witkamp, L. M. K. Vandersypen, and L. P. Kouwenhoven, "Single-shot read-out of an individual electron spin in a quantum dot," Nature, vol. 430, pp. 431-435, 2004.

[22] F. H. L. Koppens, C. Buizert, K. J. Tielrooij, I. T. Vink, K. C. Nowack, T. Meunier, L. P. Kouwenhoven, and L. M. K. Vandersypen, "Driven coherent oscillations of a single electron spin in a quantum dot," Nature, vol. 442, pp. 766-771, 2006.

[23] N. Shaji, C. B. Simmons, M. Thalakulam, L. J. Klein, H. Qin, H. Luo, D. E. Savage, M. G. Lagally, A. J. Rimberg, R. Joynt, M. Friesen, R. H. Blick, S. N. Coppersmith, and M. A. Eriksson, "Spin blockade and lifetime-enhanced transport in a few-electron $\mathrm{Si} / \mathrm{SiGe}$ double quantum dot," Nat. Phys., vol. 4, pp. 540-544, 2008.

[24] M. Piore-Ladrière, T. Obata, Y. Tokura, Y.-S. Shin, T. Kubo, K Yoshida, T. Taniyama, and S. Tarucha, "Electrically driven single-electron spin resonance in a slanting Zeeman field," Nat. Phys., vol. 4, pp. 776-779, 2008.

[25] Y.-S. Shin, T. Obata, Y. Tokura, M. Piore-Ladrière, R. Brunner, T. Kubo, K. Yoshida, and S. Tarucha, "Single-spin readout in a double quantum dot including a micromagnet," Phys. Rev. Lett., vol. 104, pp. 046802, 2010.

[26] M. G. Borsellia, K. Eng, E. T. Croke, B. M. Maune, B. Huang, R. S. Ross, A. A. Kiselev, P. W. Deelman, I. AlvaradoRodriguez, A. E. Schmitz, M. Sokolich, K. S. Holabird, T. M. Hazard, M. F. Gyure, and A. T. Hunter, "Pauli spin blockade in undoped $\mathrm{Si} / \mathrm{SiGe}$ two-electron double quantum dots," Appl. Phys. Lett., vol. 99, pp. 063109, 2011.

[27] R. Brunner, Y.-S. Shin, T. Obata, M. Piore-Ladrière, T. Kubo, K. Yoshida, T. Taniyama, Y. Tokura, and S. Tarucha, "Twoqubit gate of combined single-spin rotation and interdot spin exchange in a double quantum dot," Phys. Rev. Lett., vol. 107, pp. 146801, 2011. 
[28] L. Gaudreau, G. Granger, A. Kam, G. C. Aers, S. A. Studenikin, P. Zawadzki, M. Piore-Ladrière, Z. R. Wasilewski, and A. S. Sachrajda, "Coherent control of three-spin states in a triple quantum dot," Nat. Phys., vol. 8, pp. 54-58, 2012.

[29] Y.-S. Shin, "The average energy and molar specific heat at constant volume of an Einstein solid measured by an observer with fluctuating frame of reference," Am. J. Phys. Appl., vol. 7, pp. 21-26, 2019.
[30] S. Datta, Quantum Transport: Atom to Transistor, Cambridge University Press, New York, 2005, pp. 1-11.

[31] M. Paulsson and S. Datta, "Thermoelectric effect in molecular electronics,” Phys. Rev. B, vol. 67, pp. 241403, 2003.

[32] S. Datta, Quantum Transport: Atom to Transistor, Cambridge University Press, New York, 2005, pp. 30-31. 